\title{
Neural network based robust tracking control for nonholonomic mobile robotic system
}

\author{
Ying-Nan Bian and Jin-Zhu Peng ${ }^{\dagger}$ \\ School of electrical engineering, Zhengzhou university, \\ Zhengzhou, Henan, China \\ Corresponding author. E-mail: jzpeng@zzu.edu.cn
}

\begin{abstract}
A hybrid tracking control scheme which combines RBF neural network with nonlinear $H_{\infty}$ method is proposed. RBF neural network is designed to approximate the system uncertainty terms, and $H_{\infty}$ control is utilized to achieve a desired robust tracking performance. Based on Lyapunov theory, the tracking errors of the closed-loop system are bounded. Besides, a specified $H_{\infty}$ tracking performance is obtained by the proposed robust hybrid control even though the disturbances are merely integral bounded. Compared the proposed method with the computed torque control under the uncertainties and external disturbances, simulation experiments show the effectiveness of the proposed control strategy.

Keywords: RBF neural network; Computed torque control; $H_{\infty}$ control; Lyapunov stability.
\end{abstract}

\section{Introduction}

For the theme of tracking control for wheeled mobile robot, how to solve the nonlinear uncertainties of the system is still an active research field. In general, single control [1] scheme can usually not obtain ideal results. In recent years, hybrid control [2] strategies have been proposed in succession. Wang [3] achieved the real-time tracking target task by combining visual servoing system with the adaptive algorithm. Wong [4] stated fuzzy tracking systems based on switching grey prediction and it can be extended as a flexible strategy in dealing with the uncertain environment in robotics applications. However, the nominal inertia matrix, coriolis force matrix and gravity matrix should be precisely known in the above papers. Khalaji [5] formulated a controller which colligated robust control law and adaptive control method, it has a better tracking performance in consideration of upper-bounds of disturbances. In [6], Peng described a scheme based on fuzzy logic system and $H_{\infty}$ control methodologies. However, the disturbances assumed in the above literatures need to be uniformly bounded. 
In this paper, only the nominal inertia matrix is assumed to be known in the proposed scheme. Nonlinearity that attributes to errors of the system modeling can be perfectly approximated by RBF neural network. Moreover, the robust $H_{\infty}$ control approach can guarantee robustness to parametric and dynamics disturbances. Especially, the disturbances are assumed to be integral limited in the proposed scheme. Based on Lyapunov stability theorem, the proposed robust controller can guarantee stability of closed-loop system and a certain tracking performance. Finally, simulation examples show the efficiency of the proposed method.

\section{Robust Hybrid Control Design}

Consider a two-wheel mobile platform with nonholonomic constraints, according to reference [6][7], the kinetic equation is redefined as follows,

$$
\begin{aligned}
& \hat{M}(q) \ddot{\theta}+\widehat{C}(q, \dot{q}) \dot{\theta}+\bar{G}(q)+\bar{\tau}_{d}=\bar{\tau} \\
& \dot{\theta}=\left[\begin{array}{ll}
w_{r} & w_{l}
\end{array}\right]^{T}
\end{aligned}
$$

where $w_{r}$ and $w_{l}$ denote the angular speed of the right wheel and the left. However, the precise values of the the parameters $\bar{M}(q), \widehat{C}(q, \dot{q})$ and $\bar{G}(q)$ in the robot dynamic model are difficult to acquire since measuring errors, environment. Therefore, it is assumed that real values can be separated as nominal parts defined by $\widehat{M}_{0}(q), \widehat{C}_{0}(q, \dot{q})$ and $\bar{G}_{0}(q)$ and uncertain parts denoted by $\Delta \widehat{M}(q), \Delta \widehat{C}(q, \dot{q})$ and $\Delta \bar{G}(q)$ respectively. Then we get a new equation,

$$
\begin{aligned}
& \widehat{M}_{0}(q) \ddot{\theta}+\mathrm{T}\left(x_{e}\right)+\bar{\tau}_{d}=\bar{\tau} \\
& \mathrm{T}\left(x_{e}\right)=\Delta \hat{M}(q) \ddot{\theta}+\widehat{C}(q, \dot{q}) \dot{\theta}+\bar{G}(q)
\end{aligned}
$$

It is assumed that the nominal parts of the inertia matrix in the mobile robotic system is precisely known, other uncertain parts are unknown. At the same time, RBF neural network can be applied to approximate $\mathrm{T}\left(x_{e}\right)=W^{* T} H(\bullet)+\varepsilon$, where $W^{*}$ represents the optimal weight matrix, $H(\bullet)$ denotes primary function of the RBF neural network, $\varepsilon$ stands for the approximation error of the network. We assume that there exists $\Omega_{W}$ and the ideal weights are in the compact set $\Omega_{W}$, which is defined as $\Omega_{W}=\left\{W \in R^{m \times n}:\|W\| \leq M_{W}\right\}$. The optimal weight can be defined as $W^{*}=\arg \min \left\{\sup \left|\mathrm{T}\left(x_{e}\right)-W^{T} H(\bullet)\right|\right\}$. According to the mobile robot system, the proposed control law is as follows, 


$$
\bar{\tau}=\widehat{M}_{0}(q)\left(\ddot{\theta}_{d}+K_{v} \dot{e}+K_{p} e\right)+W^{T} H(\bullet)+u(3)
$$

where $u$ is used to compensate the errors of external disturbance and neural network approximation in the system. Substitute Eq. (3) into Eq. (2),

$$
\widehat{M}_{0}(q)\left(\ddot{e}+K_{v} \dot{e}+K_{p} e\right)=\varepsilon-u+\tilde{W}^{T} H(\bullet)+\bar{\tau}_{d}
$$

where $\tilde{W}=W^{*}-W$ denotes error of weight matrix. Then we define the state vector as $x=\left[\begin{array}{ll}x_{1}^{T} & x_{2}^{T}\end{array}\right]^{T}=\left[\begin{array}{ll}e^{T} & \dot{e}^{T}\end{array}\right]^{T}$. Easily, the following equation can be calculated,

$$
\dot{x}=A x+B \widehat{M}_{0}^{-1}(q)\left(\tilde{W}^{T} H(\bullet)+\varepsilon+\bar{\tau}_{d}-u\right)
$$

where $A=\left[\begin{array}{cc}0 & I_{n \times n} \\ -K_{p} & -K_{v}\end{array}\right], B=\left[\begin{array}{c}0 \\ I_{n \times n}\end{array}\right]$.

Assumption 1. It is assumed that the error of the approximation in the RBF neural network belongs to the external disturbance. Then we defined $\delta=\widehat{M}_{0}^{-1}(q)\left(\varepsilon+\bar{\tau}_{d}\right)$ as the total external disturbance. Besides, $\delta>0$ and its energy integral is less than a positive $\sigma_{d}$.

Thereom 1. Considering the robotic system dynamic Eq. (5), there exists a matrix $P=P^{T}>0$ such that the following matrix equality holds

$$
P A+A^{T} P+P B\left(\frac{1}{\gamma^{2}} I_{n \times n}-2 R^{-1}\right) B^{T} P=-Q
$$

where $R=R^{T}>0$ and $Q=Q^{T}>0$ is positive definite symmetric matrix, $I_{n \times n}$ is identity matrix, $\gamma>0$ denotes prescribed disturbance attenuation index. Define

$$
u=\widehat{M}_{0}(q) R^{-1} B^{T} P x, \dot{W}=\Gamma^{-1} H(\cdot) x^{T} P B \widehat{M}_{0}^{-1}(q)
$$

where $\Gamma$ is positive definite diagonal gain matrix.

Proof. Select a Lyapunov function candidate, 


$$
\begin{aligned}
& L=\frac{1}{2} x^{T} P x+\frac{1}{2} \operatorname{tr}\left(\tilde{W}^{T} \Gamma \tilde{W}\right) \\
& \dot{L}=\frac{1}{2}\left[A x+B \hat{M}_{0}^{-1}(q)\left(\tilde{W}^{T} H(\cdot)-u+\varepsilon+\bar{\tau}_{d}\right)\right]^{T} P x+\frac{1}{2} x^{T} P\left[A x+B \hat{M}_{0}^{-1}(q)\left(\tilde{W}^{T} H(\cdot)-u+\varepsilon+\bar{\tau}_{d}\right)\right] \\
& +\operatorname{tr}\left(\tilde{W}^{T} \Gamma \dot{W}\right) \\
& =\frac{1}{2} x^{T}\left(P A+A^{T} P+P B\left(\frac{I_{n \times n}}{\gamma^{2}}-2 R^{-1}\right) B^{T} P\right) x-\frac{1}{2}\left(\frac{1}{\gamma} B^{T} P x-\gamma \delta\right)^{T}\left(\frac{1}{\gamma} B^{T} P x-\gamma \delta\right)+\frac{1}{2} \gamma^{2} \delta^{T} \delta \\
& \leq-\frac{1}{2} x^{T} Q x+\frac{1}{2} \gamma^{2} \delta^{T} \delta
\end{aligned}
$$

Integrating the above inequality from $\mathrm{t}=0$ to $\mathrm{t}=\mathrm{T}$ yields,

$$
L(x(T), \tilde{W}(T))-L(x(0), \tilde{W}(0)) \leq-\frac{1}{2} \int_{0}^{T} x^{T}(t) Q x(t) d t+\frac{\gamma^{2}}{2} \int_{0}^{T} \delta^{T}(t) \delta(t) d t(8)
$$

Since $L(x(T), \tilde{W}(T)) \geq 0$ the above inequality leads to

$$
\int_{0}^{T}\|x(t)\|_{Q}^{2} d t \leq 2 L(x(0), \tilde{W}(0))+\gamma^{2} \int_{0}^{T}\|\delta(t)\|^{2} d t
$$

As a result, the $H_{\infty}$ tracking performance can be achieved. According to Assumption1, the $H_{\infty}$ tracking performance of total system can be satisfied. Next we can deduce further as follows,

$$
\dot{L} \leq-\frac{1}{2} \lambda_{\min }(Q)\|x(t)\|^{2}+\frac{1}{2} \gamma^{2} \sigma_{d}^{2}
$$

where $\lambda_{\min }(Q)$ represent the minimum eigenvalue of $\mathrm{Q}$ matrix. Then we choose suitable $\lambda_{\min }(Q)$, there exists $\zeta>0$ that makes the following formula correct,

$$
\dot{L} \leq-\zeta\|x(t)\|^{2}<0, \forall\|x(t)\|>\varepsilon
$$

where $\varepsilon$ is a arbitrarily small positive number. As a result, all the state variables in the closed loop system are bound according to our analysis.

\section{Simulation Result}

Simulations are carried out in two-wheel robot platform .The nominal parameters are $\mathrm{m}=10 \mathrm{~kg}, \mathrm{R}=0.25 \mathrm{~m}, \mathrm{r}=0.05 \mathrm{~m}$ and $\mathrm{d}=0$. It is assumed that the desire trajectories of $\theta_{r d}, \theta_{l d}$ is a trapezoid broken line. The initial line is $\theta_{r d}=t+0.1 \cos t, \theta_{l d}=t$.The initial conditions are $\theta(0)=\left[\begin{array}{ll}1 & 1\end{array}\right]^{T}$ and 
$\dot{\theta}(0)=\left[\begin{array}{ll}0 & 0\end{array}\right]^{T}$.Select controller parameters $K_{v}=50 I_{2 \times 2}, \quad K_{p}=150 I_{4 \times 4}$, $\gamma=0.2, Q=40 I_{4 \times 4}, R=0.001 I_{2 \times 2}, \bar{\tau}_{d}=\left[10(\sin t) e^{-0.2 t}, 10(\cos t) e^{-0.2 t}\right]^{T}$. The number of hidden layer neurons is selected as 7. Compared the CTC method with the proposed method, the Fig.1, Fig.2 show the results of the simulation experiments.

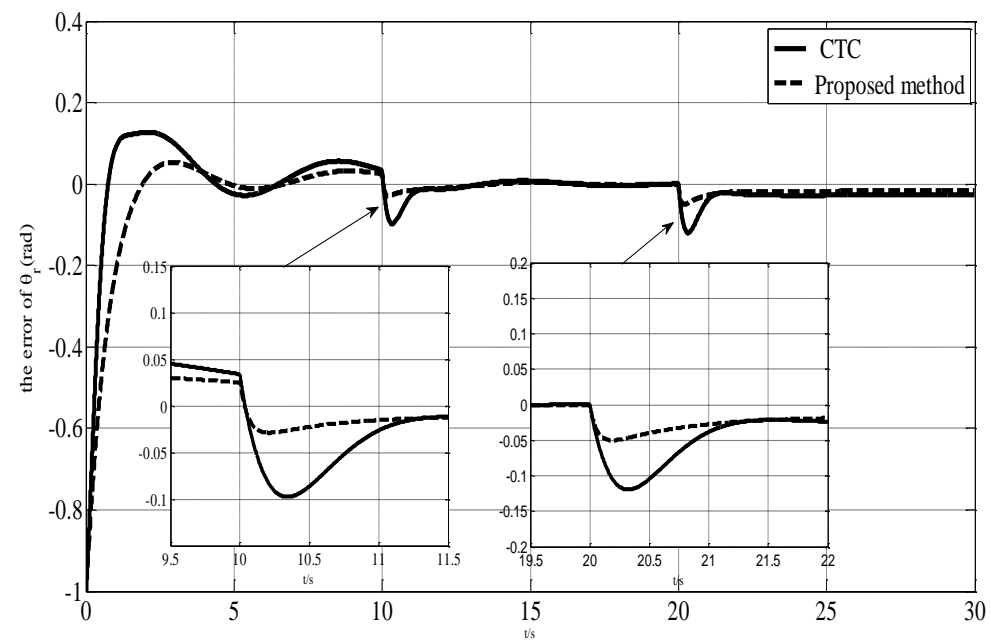

Fig.1. The tracking error of right wheel

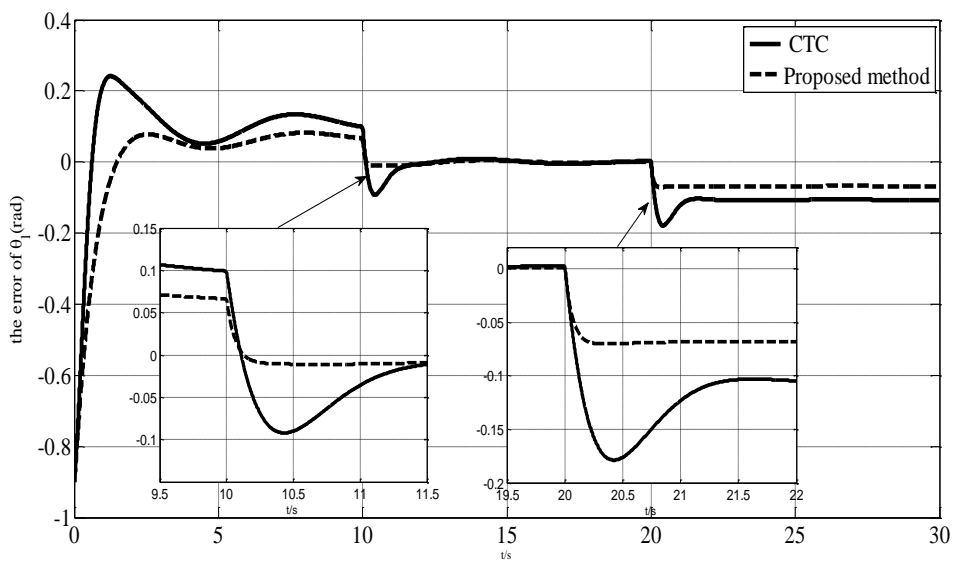

Fig.2. The tracking error of left wheel

From the above figures, It is obviously that the tracking error of the proposed method is better than the CTC method. Besides, the chattering of tracking error has been remarkably reduced when the tracking trajectory changes at $t=10 \mathrm{~s}$ and $t=20 \mathrm{~s}$. As a result, we can conclude that the robust hybrid 
controller has ideal tracking performance and improve the robustness of the system.

\section{Conclusion}

This paper presents a robust tracking control scheme for mobile robotic platform with mathematical derivations of global stability. The central idea is to combine RBF neural network with a robust $H_{\infty}$ controller. Theoretical analysis shows that robustness to approximation errors can be ensured and the effect of finite-energy, immeasurable external disturbances entering the system can be attenuated. Moreover, It can be guaranteed that all signals in the closed loop are bounded. The results of the simulation demonstrate that the proposed control method has better robust performance than CTC method under the same external disturbances.

\section{Acknowledgement}

This work partially supported by Outstanding Young Talent Research Fund of Zhengzhou University(1521319001) and Henan Provincial Natural Science Foundation.

\section{Reference}

1. ORTEGA R, SPONG M W. Adaptive motion control of rigid robot: $a$ tutorial [J]. (Automatica, 1989).

2. Spandan Roy, Nandy, S, Ray, Retal. Time Delay Sliding Mode Control of Nonholonomic Wheeled Mobile Robot: Experimental Validation, IEEE International Conference on Robotics and Automation.(Hong Kong, 2014).

3. Wang, K, Liu, YH. Visual Servoing Trajectory Tracking of Nonholonomic Mobile Robots Without Direct Position Measurement. IEEE TRANSACTIONS ON ROBOTICS. (Shatin, Hong Kong, 2014).

4. Ching-Chang Wong. Fuzzy tracking method with a switching grey prediction for mobile robot. 10th IEEE International Conference on Fuzzy Systems. (MELBOURNE, AUSTRALIA, 2001).

5. Khalaji. Robust Adaptive Controller for a Tractor-Trailer Mobile Robot. (IEEE-ASME TRANSACTIONS ON MECHATRONICS. JUN 2014).

6. Jinzhu Peng. Robust Tracking Control for Robotic Manipulator via Fuzzy Logic System and $H_{\infty}$ Approaches. (Journal of Control Science Engineering, 2015).

7. Mee-Seub Lim. A Hybrid System Approach to Motion Control of Wheeled Mobile Robots. (Canada, 1998). 